\title{
Analysis of microarray data for identification of key microRNA signatures in glioblastoma multiforme
}

\author{
SANU K. SHAJI, DAMU SUNILKUMAR, N.V. MAHALAKSHMI, GEETHA B. KUMAR and BIPIN G. NAIR
}

School of Biotechnology, Amrita Vishwa Vidyapeetham, Kollam, Kerala 690525, India

Received December 15, 2018; Accepted June 6, 2019

DOI: $10.3892 / 01.2019 .10521$

\begin{abstract}
Glioblastoma multiforme (GBM) is one of the most malignant types of glioma known for its reduced survival rate and rapid relapse. Previous studies have shown that the expression patterns of different microRNAs (miRNA/miR) play a crucial role in the development and progression of GBM. In order to identify potential miRNA signatures of GBM for prognostic and therapeutic purposes, we downloaded and analyzed two expression data sets from Gene Expression Omnibus profiling miRNA patterns of GBM compared with normal brain tissues. Validated targets of the deregulated miRNAs were identified using MirTarBase, and were mapped to Search Tool for the Retrieval of Interacting Genes/Proteins, Database for Annotation, Visualization and Integrated Discovery and Kyoto Encyclopedia of Genes and Genomes databases in order to construct interaction networks and identify enriched pathways of target genes. A total of 6 miRNAs were found to be deregulated in both expression datasets studied. Pathway analysis demonstrated that most of the target genes were enriched in signaling cascades connected to cancer development, such as 'Pathways in cancer', 'Focal adhesion' and 'PI3K-Akt signaling pathway'. Of the five target genes that were enriched in the glioblastoma pathway, in the WikiPathway database, both HRas proto-oncogene, GTPase and MET proto-oncogene, receptor tyrosine kinase target genes of hsa-miR-139-5p, were found to be significantly associated with patient survival. The present study may thus form the basis for further exploration of hsa-miR-139-5p, not only as a therapeutic agent, but also as a diagnostic biomarker for GBM as well as a predictive marker for patient survival.
\end{abstract}

Correspondence to: Dr Bipin G. Nair, School of Biotechnology, Amrita Vishwa Vidyapeetham, Amritapuri, Clappana P.O, Kollam, Kerala 690525, India

E-mail: bipinnair03@gmail.com

Key words: glioblastoma multiforme, gene expression omnibus, microRNA, GEO2R, pathway analysis

\section{Introduction}

Glioblastoma multiforme (GBM) is the most common and malignant types of glioma, accounting for the major cause of brain cancer death, with a median patient survival of about 15 months from diagnosis (1). It is considered to be the 12th leading cause of cancer-associated death in the USA (2). Treatment of GBM includes surgery, chemotherapy and radiation therapy, but resistance to chemotherapeutic agents and a high frequency of relapse after surgery, pose difficulties in the therapeutic intervention of this disease (3-5). The molecular determinants of GBM are not well understood even though there have been advances in GBM research in last few decades. It is therefore important to identify and establish a clear mechanism of GBM onset and progression that may help in early diagnosis, as well as development of new strategies for combating this disease.

Even though microRNAs (miRNAs) were discovered in 1993 (6), their involvement in cancer was first reported only in 2002 (7). MicroRNAs are small non-coding RNAs ranging from $17-25 \mathrm{bp}$, which are involved in the post-translational regulation of gene expression by the partial complementarity of binding sites in the $3^{\prime}$ untranslated regions of mRNA (8). MicroRNAs can regulate gene expression either by mRNA cleavage or by translational repression (9). Some miRNAs can act as tumour suppressors by downregulating oncogenes, whereas others called oncomirs, act to promote tumorigenesis, by lowering expression levels of tumour suppressor genes (10). According to MirBase, there are 1,917 precursors and 2,654 mature miRNAs in humans, each miRNA being able to regulate the expression of several mRNAs. Each of these mRNAs are in turn regulated by different miRNAs, implicating the presence of a very complex regulatory machinery which demands a focused study in order to map mRNAs and its miRNA regulators. Deregulated miRNA expression patterns have been observed in a wide variety of human malignancies, such as pancreas, breast, colon, lung and skin cancers (11).

Even though there are ongoing efforts to establish miRNA patterns in GBM models, identification of new biomarkers that could be used for diagnostic and prognostic purposes is a very significant unmet need. Therefore, the current study is focused on investigating the GBM miRNA expression profile data sets collected from GEO database, followed by identification of differentially expressed miRNAs between normal and GBM tissues. Furthermore, the present study also details the 
Table I. Most significantly upregulated and downregulated miRNAs.

\begin{tabular}{|c|c|c|c|c|}
\hline \multirow[b]{2}{*}{ miRNA ID } & \multicolumn{2}{|c|}{$\log \mathrm{FC}$} & \multicolumn{2}{|c|}{ adj.P.Val } \\
\hline & GSE65626 & GSE25631 & GSE65626 & GSE25631 \\
\hline hsa-miR-138-2-3p & -4.983773 & -3.540977 & 0.000943 & 0.001108 \\
\hline hsa-miR-139-3p & -6.720897 & -3.16659 & 0.012132 & 0.009653 \\
\hline hsa-mir-139-5p & -4.09792 & -1.469392 & 0.039613 & 0.025831 \\
\hline hsa-miR-338-5p & -7.989224 & -3.3562 & 0.013202 & 0.001108 \\
\hline hsa-miR-770-5p & -4.504915 & -2.807833 & 0.027344 & 0.039478 \\
\hline
\end{tabular}

$\operatorname{logFC}, \log$ fold change; adj.P.Val, Adjusted P-Value; miRNA/miR, microRNA.

exploration of their targets, and construction of their target gene interaction networks, to get a better understanding of the underlying mechanism of GBM development and progression.

\section{Materials and methods}

Microarray data. The GBM miRNA expression data from GEO dataset with accession number GSE65626 and GSE25631 were selected for the analysis. GSE65626 contained both gene expression and miRNA expression analysis of 3 primary GBM tissues which were compared with normal adjacent tissues. The miRNA expression data performed based on the GPL19117 platform (Affymetrix Multispecies miRNA-4 Array), was taken for the current analysis. GSE25631 contained the expression data of 82 primary GBM and 5 normal brain tissues, performed in a platform based on GPL8179 Illumina Human v2 MicroRNA expression beadchip.

Microarray data processing. GEO2R (http://www.ncbi. nlm.nih.gov/geo/geo2r), is an online tool which compares two groups of samples in GEO dataset in order to identify deregulated miRNAs in GBM samples, compared to adjacent normal tissues. The two packages of Bioconductor project in $\mathrm{R}$ platform, GEOquery and limma, enable accurate analysis of processed microarray data supplied, to detect deregulated miRNAs from GEO datasets. False discovery rate (FDR) was minimized by applying adjusted P-values (adj P-value) based on Benjamini-Hochberg method. The llog Fold Change $(\log F C) \mid>1$ and adj P-value $<0.05$ were taken as cut off values for detection of upregulated or downregulated miRNAs in the samples.

Identification of targets of deregulated miRNAs. Validated target genes of deregulated miRNAs were determined using MirTarbase (http://mirtarbase.mbc.nctu.edu.tw), which is a database of manually curated miRNA target gene interactions, obtained from the literature. The target genes which are tagged with strong evidence (Western blot/Reporter assay/Quantitative real-time PCR) are selected for further analysis.

Gene ontology $(G O)$ and pathway analysis. The Database for Annotation, Visualization and Integrated Discovery (DAVID) (http://david.ncifcrf.gov/), is a web tool for high throughput analysis of gene function, obtained from genomic experiments. In the present study, DAVID was used to perform gene ontology (GO) and Kyoto Encyclopedia of Genes and Genomes (KEGG) pathway analysis. GO categorizes ontology terms into molecular function, cellular components and biological processes. KEGG pathway database analysis was performed to identify enrichment of deregulated miRNA target genes, which could be mapped to specific pathways. Both the GO and KEGG pathway analysis were performed with a strict criterion for selection of significant enrichment (False Discovery Rate (RDF) <0.05). Wikipathway analysis and mapping of target genes were done using a Cytoscape 3.6 plugin (WikiPathways), and is visualized in Cytoscape 3.6.

PPI network analysis and clusters selection. Protein interaction network (PPI) of the target genes were constructed using Search Tool for the Retrieval of Interacting Genes/Proteins (STRING, http://string.embl.de/), which is a database of predicted and known protein-protein interactions. The PPI network was created with a high confidence score (confidence score $\geq 7$ ) and was visualized by Cytoscape 3.6 software. In order to detect functional modules in the network, a Cytoscape plugin, Molecular Complex Detection (MCODE) was used. An MCODE score greater than 3, and the total number of nodes greater than 4 , was kept as cut-off criteria.

Kaplan-Meier survival curves analysis. The survival analysis was performed using OncoLnc (http://www.oncolnc.org), an online data mining tool which uses survival data from cancer studies executed by The Cancer Genome Atlas (TCGA). Patients were divided into high expression and low expression groups based on a 25 th percentile cut off.

\section{Results}

Identification of deregulated miRNAs. In this study, we obtained two publically available miRNA microarray data sets, GSE65626 and GSE25631, from GEO. GSE65626 contains a total of 12 samples, in which 3 primary GBM tissues are compared with their normal adjacent tissues in duplicates. GSE25631 has the miRNA expression profiles of 5 normal brain tissues and 82 primary GBM tissues. A total of 11 and 23 upregulated miRNAs were observed in 

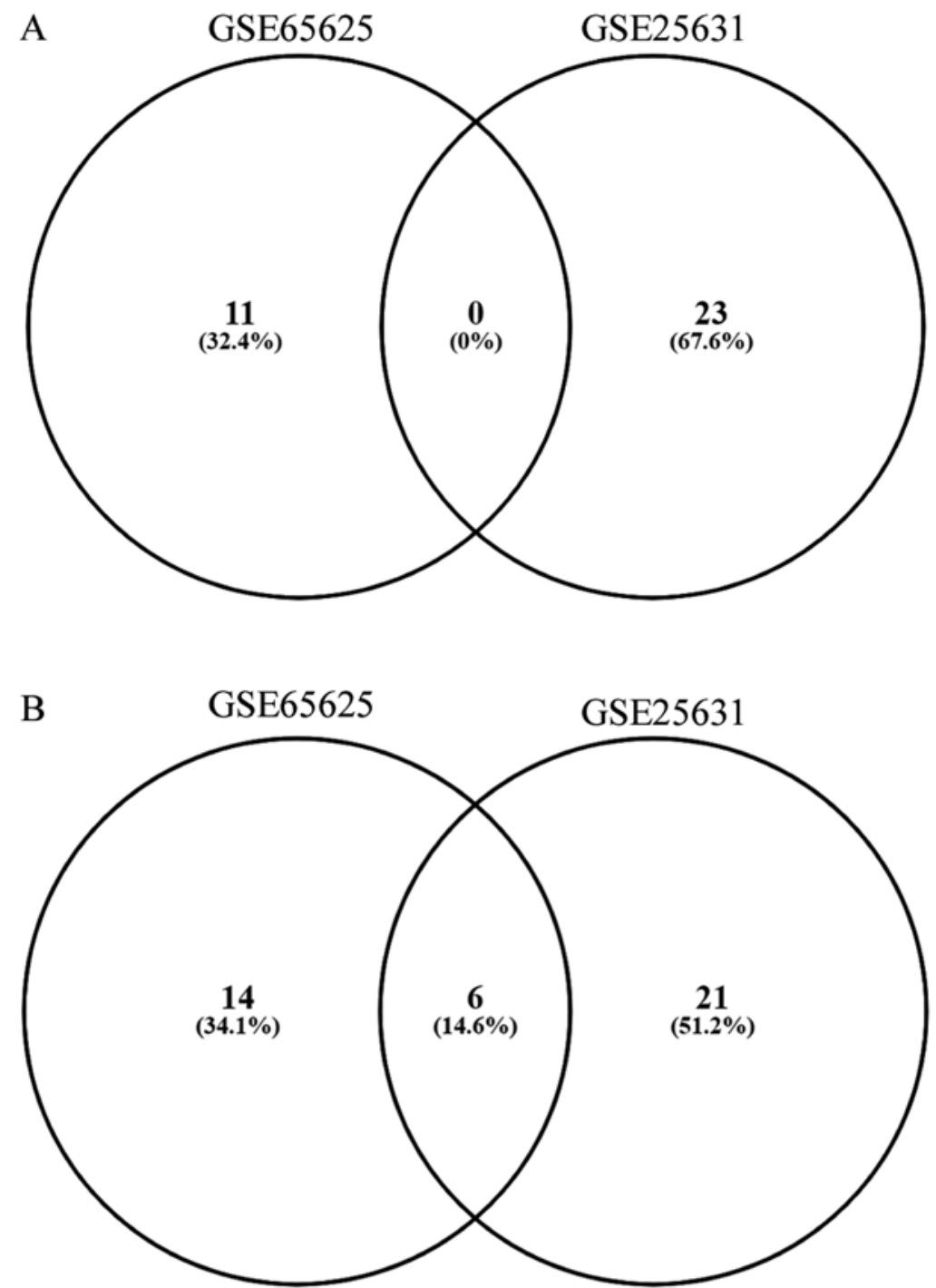

Figure 1. Deregulated miRNAs in the datasets. (A) Upregulated miRNAs. A total of 11 and 23 upregulated miRNAs were identified in GSE65626 and GSE25631, respectively. None of the upregulated miRNAs were commonly present in both the datasets. (B) Downregulated miRNAs. A total of 14 and 21 miRNAs were downregulated in GSE65626 and GSE25631, respectively. A total of 6 miRNAs were downregulated in both the datasets analyzed. miRNAs, microRNAs.

GSE65626 and GSE25631 respectively (Fig. 1A). However, none of the upregulated miRNAs were found to show similar expression trends in both the datasets. On other hand, of the 20 and 27 down-regulated miRNAs present in GSE65626 and GSE25631 respectively, 6 (hsa-miR-139-3p, hsa-miR-139-5p, hsa-miR-138-2-3p, hsa-miR-338-5p, hsa-miR-383-5p and hsa-miR-770-5p) were found to be expressed in reduced levels in GBM tissues as compared to adjacent normal brain tissues in both the datasets (Fig. 1B; Table I). Among these miRNAs, hsa-miR-338-5p was found to show the highest average downregulation $(\log \mathrm{FC}=-5.672712)$, whereas hsa-mir-139-5p showed the least average downregulation $(\log \mathrm{FC}=-2.783656)$ in both the datasets.

Identification of the miRNA gene regulatory network. Since miRNAs play a very important role in the regulation of post-translational gene expression, we queried the targets of all 6 deregulated miRNAs (hsa-miR-139-3p, hsa-miR-139-5p, hsa-miR-138-2-3p, hsa-miR-338-5p, hsa-miR-383-5p and hsa-miR-770-5p) from miRTarBase, which is a database of experimentally validated miRNA-target gene interactions. Altogether 49 interactions were obtained with strong experimental evidence (Western blot/Reporter assay/Quantitative real-time PCR), for 5 out of 6 miRNAs, which were visualized using Cytoscape (Fig. 2). Amongst the deregulated miRNAs, hsa-miR-138-2-3p was not found to have any validated target gene available in the database, in contrast to the other miRNAs, namely, hsa-miR-139-3p, hsa-miR-139-5p, hsa-miR-338-5p, hsa-miR-383-5p and hsa-miR-770-5p, which have 3, 26, 7, 10 and 3 target genes respectively. IGF1R is a target of both hsa-miR-139-5p and hsa-miR-383-5p whereas MMP11 is targeted by both hsa-miR-139-3p, hsa-miR-139-5p.

Gene ontology (GO) functional annotation and pathway enrichment of targets genes. To gain insights into the biological pathways of target genes, we performed GO categories and pathway enrichment analysis using DAVID software, a web-accessible program for the functional annotation of genes obtained from genomic experiments. The list of genes were analyzed for enrichment in different GO terms, which were 


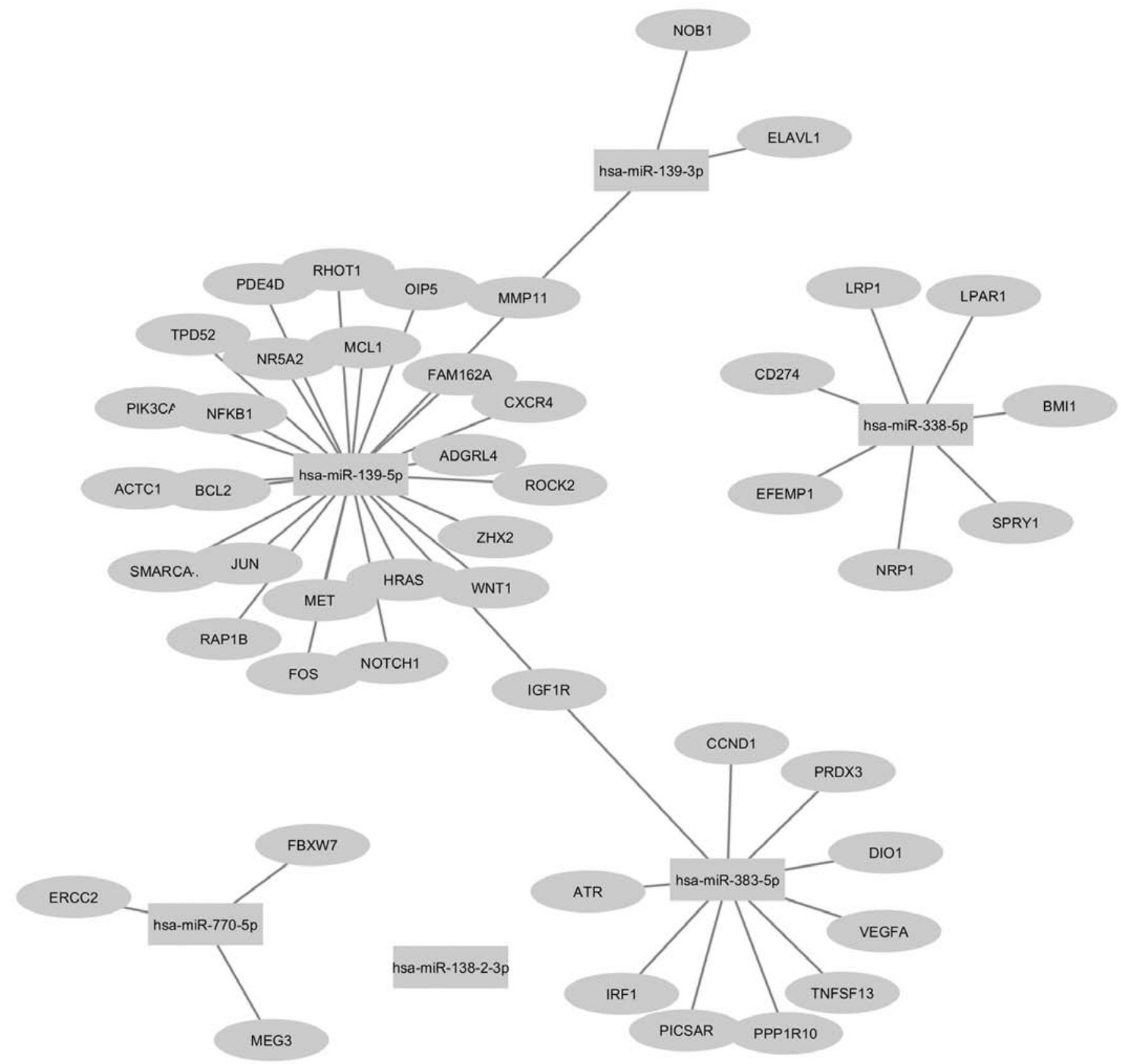

Figure 2. Deregulated miRNAs and their validated targets obtained from MirTarBase. A total of 49 genes were identified as the validated targets of deregulated miRNAs which were mapped to their respective miRNAs and visualized in Cytoscape. Boxes represent miRNAs and ovals represent genes. miRNAs, microRNAs.

grouped into sub-ontologies, namely, biological process (BP), cellular component (CC) and molecular function (MF). The minimum number of genes for the $\mathrm{GO}$ categorization was set to 2. The significant GO term for the target genes is listed in Table II. Only 7 GO terms were enriched with a selection criterion of FDR $<0.05$. Based on this, we identified that the majority of the genes in CC category are enriched in the cytosol (24 genes) and nucleoplasm (20 genes). In BP ontology, majority of the genes were enriched in positive regulation of DNA-templated transcription (10 genes), followed by negative regulation of neuron apoptotic process (6 genes), response to cytokine (5 genes) and negative regulation of anoikis (4 genes). However, in MF ontology, only one category was significantly enriched, namely, protein hetero-dimerization activity (9 genes).
Furthermore, the KEGG pathway analysis to identify the significant pathways enriched in target gene list was performed using DAVID software. With a cut-off criteria of FDR $<0.05$, only 11 pathways were obtained (Table III). The major pathway which was significantly enriched included pathways in cancer with the highest number of target genes (14 genes), followed by focal adhesion (10 genes), PI3K-Akt signaling (10 genes), microRNAs in cancer ( 9 genes). Pathways in cancer involves different signaling pathways such as Wnt, p53, VEGF, calcium, HIF-1, PPAR, Notch, PI3K-Akt, MAPK, Estrogen, cAMP, TGF beta, Hedgehog, Jak-STAT and mTOR pathways, which play critical roles in invasion and metastasis, cell proliferation, adhesion, angiogenesis, apoptosis and resistance to chemotherapy. Focal adhesion pathway plays a crucial role in cell motility, cell survival and cell proliferation. PI3K-Akt 
Table II. Top enriched GO terms.

A, Cellular component

\begin{tabular}{lcll}
\hline GO ID and term & Count & FDR & \multicolumn{1}{c}{ Genes } \\
\hline GO:0005829: cytosol & 22 & 0.008208 & HRAS, ACTC1, NRP1, MCL1, ROCK2, NOB1, \\
& & & ELAVL1, NFKB1, TNFSF13, PDE4D, PRDX3, FOS, \\
& & & SPRY1, NOTCH1, FBXW7, CCND1, JUN, BCL2, \\
& & & IRF1, RHOT1, PIK3CA, RAP1B \\
GO:0005654: nucleoplasm & 20 & 0.010017 & BMI1, MCL1, ZHX2, NOB1, ELAVL1, PPP1R10, \\
& & & NFKB1, TNFSF13, ATR, FOS, SPRY1, NOTCH1, \\
& & & FBXW7, CCND1, OIP5, JUN, IRF1, NR5A2, ERCC2, \\
& & SMARCA4
\end{tabular}

B, Biological process

\begin{tabular}{lccl}
\hline GO ID and term & Count & FDR & \\
\hline $\begin{array}{l}\text { GO:0045893: positive regulation of } \\
\text { transcription, DNA-templated }\end{array}$ & 10 & 0.009448 & WNT1, FOS, NOTCH1, JUN, IRF1, MEG3, NFKB1, \\
GO:0034097: response to cytokine & & & NR5A2, SMARCA4, ERCC2 \\
GO:2000811: negative regulation of anoikis & 4 & 0.017246 & NOTCH1, MCL1, BCL2, PIK3CA \\
$\begin{array}{l}\text { GO:0043524: negative regulation of neuron } \\
\text { apoptotic process }\end{array}$ & 6 & 0.036615 & HRAS, NRP1, LRP1, JUN, BCL2, PIK3CA \\
\hline
\end{tabular}

C, Molecular function

\begin{tabular}{lccl}
\hline GO ID and term & Count & FDR & \multicolumn{1}{c}{ Genes } \\
\hline GO:0046982: protein heterodimerization activity & 9 & 0.028328 & $\begin{array}{l}\text { FOS, NOTCH1, MCL1, JUN, BCL2, VEGFA, ZHX2, } \\
\text { NFKB1, TPD52 }\end{array}$ \\
\hline
\end{tabular}

FDR, false discovery rate; GO, Gene Ontology.

Table III. Enriched Kyoto Encyclopedia of Genes and Genomes pathways of the target genes.

\begin{tabular}{|c|c|c|c|}
\hline Term & Count & FDR & Genes \\
\hline Pathways in cancer & 14 & $5.10 \times 10^{-6}$ & $\begin{array}{l}\text { HRAS, ROCK2, MET, NFKB1, LPAR1, IGF1R, WNT1, FOS, CCND1, } \\
\text { CXCR4, BCL2, JUN, VEGFA, PIK3CA }\end{array}$ \\
\hline Focal adhesion & 10 & $2.10 \times 10^{-4}$ & $\begin{array}{l}\text { IGF1R, HRAS, CCND1, ROCK2, JUN, BCL2, VEGFA, MET, PIK3CA, } \\
\text { RAP1B }\end{array}$ \\
\hline Renal cell carcinoma & 6 & 0.010445 & HRAS, JUN, VEGFA, MET, PIK3CA, RAP1B \\
\hline HTLV-I infection & 9 & 0.014093 & WNT1, FOS, HRAS, CCND1, NRP1, JUN, PIK3CA, NFKB1, ATR \\
\hline PI3K-Akt signaling pathway & 10 & 0.015521 & $\begin{array}{l}\text { IGF1R, HRAS, CCND1, MCL1, BCL2, VEGFA, MET, PIK3CA, NFKB1, } \\
\text { LPAR1 }\end{array}$ \\
\hline Prolactin signaling pathway & 6 & 0.016156 & FOS, HRAS, CCND1, IRF1, PIK3CA, NFKB1 \\
\hline Proteoglycans in cancer & 8 & 0.026297 & IGF1R, WNT1, HRAS, CCND1, ROCK2, VEGFA, MET, PIK3CA \\
\hline MicroRNAs in cancer & 9 & 0.030795 & BMI1, NOTCH1, HRAS, CCND1, MCL1, BCL2, VEGFA, MET, NFKB1 \\
\hline Hepatitis B & 7 & 0.043312 & FOS, HRAS, CCND1, JUN, BCL2, PIK3CA, NFKB1 \\
\hline Prostate cancer & 6 & 0.046072 & IGF1R, HRAS, CCND1, BCL2, PIK3CA, NFKB1 \\
\hline
\end{tabular}

FDR, false discovery rate. 


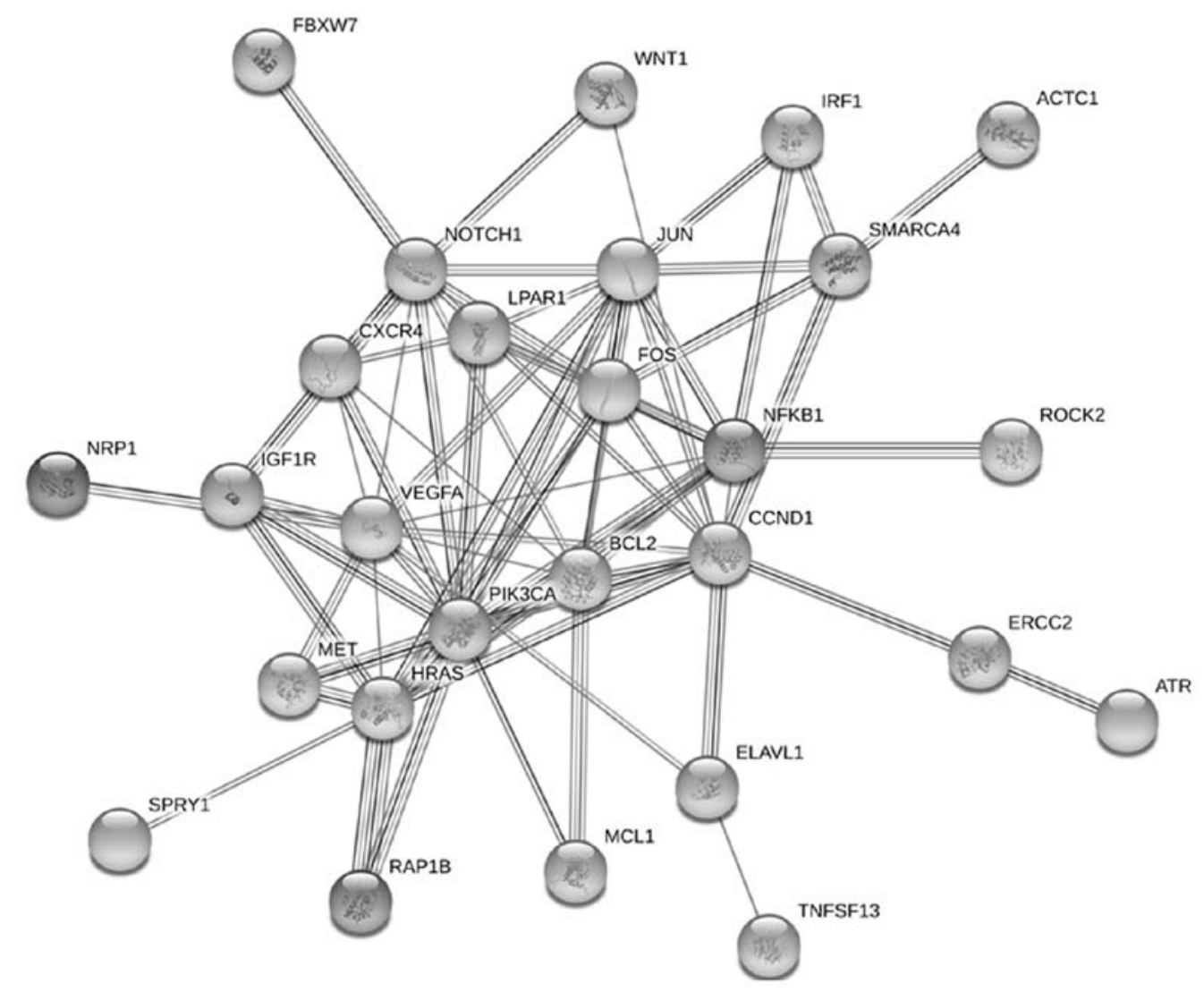

Figure 3. Protein-protein interaction network of deregulated miRNA target genes obtained from the Search Tool for the Retrieval of Interacting Genes/Proteins database. There are 44 nodes with 136 edges with an average node degree of 6.18. The analysis showed that target genes of deregulated miRNAs are biologically connected with a P-value of $4.62 \times 10^{-12}$. miRNA, microRNA.

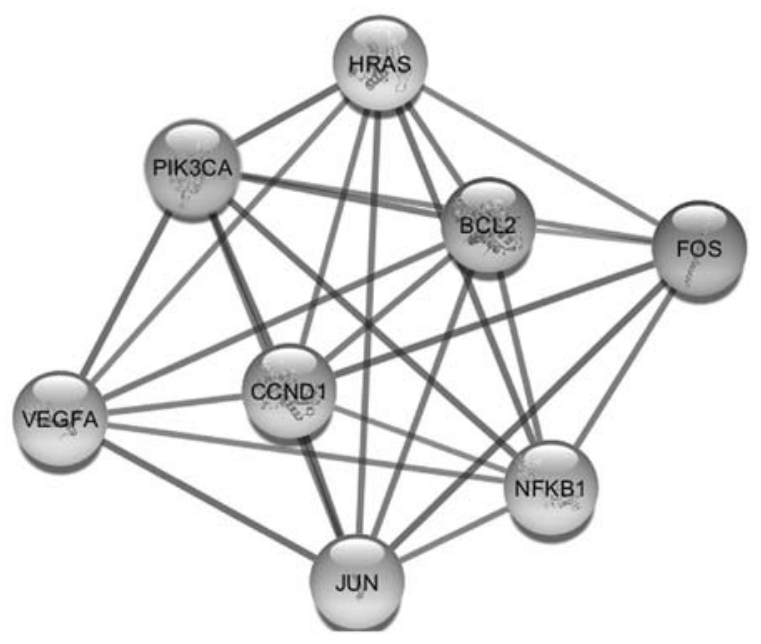

Figure 4. Only significant module found by MCODE analysis with cut-off criteria of MCODE score $>3$ and node $>4$. This module consists of 8 nodes (BCL-2, CCND1, FOS, HRAS, JUN, NFKB1, PIK3CA and VEGFA) and 27 edges, and VEGF is the seed gene in this module with an MCODE score of 6. MCODE, Molecular Complex Detection; CCND1, cyclin D1; FOS, Fos proto-oncogene, AP-1 transcription factor subunit; HRAS, HRas proto-oncogene, GTPase; NFKB1, nuclear factor $\kappa$ B subunit 1; PIK3CA phosphatidylinositol-4,5-biphosphate 3-kinase catalytic subunit $\alpha$; VEGFA, vascular endothelial growth factor A.

signaling plays a vital role in the development and progression of GBM. Pathway enrichment analysis thus shows a significant role for these deregulated miRNAs in GBM development.
PPI network construction of DE-miRNA target genes and module analysis. In order to understand the interaction among target genes, PPI network was constructed using STRING database with a criterion of minimum required interaction score set to high confidence (0.7). There were 44 nodes with 136 edges with an average node degree of 6.18 (Fig. 3). PPI enrichment showed that target genes of deregulated miRNAs are at least partially biologically connected (P-value:4.62 $\mathrm{e}^{-12}$ ). The PPI interaction network was further explored for identification of clusters using a Cytoscape based plug in, MCODE. An MCODE score greater than 3, and the total number of nodes greater than 4 , was kept as cut-off criteria for identification of functional modules. Only a single cluster was identified by MCODE which consisted of 8 nodes (BCL-2, CCND1, FOS, HRAS, JUN, NFKB1, PIK3CA, VEGFA) and 27 edges (Fig. 4). Furthermore, VEGFA has been identified as the seed gene in this cluster with an MCODE score of 6.

Mapping of target genes to glioblastoma pathway in wikipathway. We downloaded signaling pathways in glioblastoma from Wikipathway which included most frequently altered genes in GBM, drawn together from literature and public database resources, using plug-in Wikipathways, and visualized them in Cytoscape, followed by mapping of deregulated miRNA targets genes. Our analysis showed that five of the target genes, namely, CCND1, IGF1R, PIK3CA, HRAS and MET play a significant role in GBM signaling 


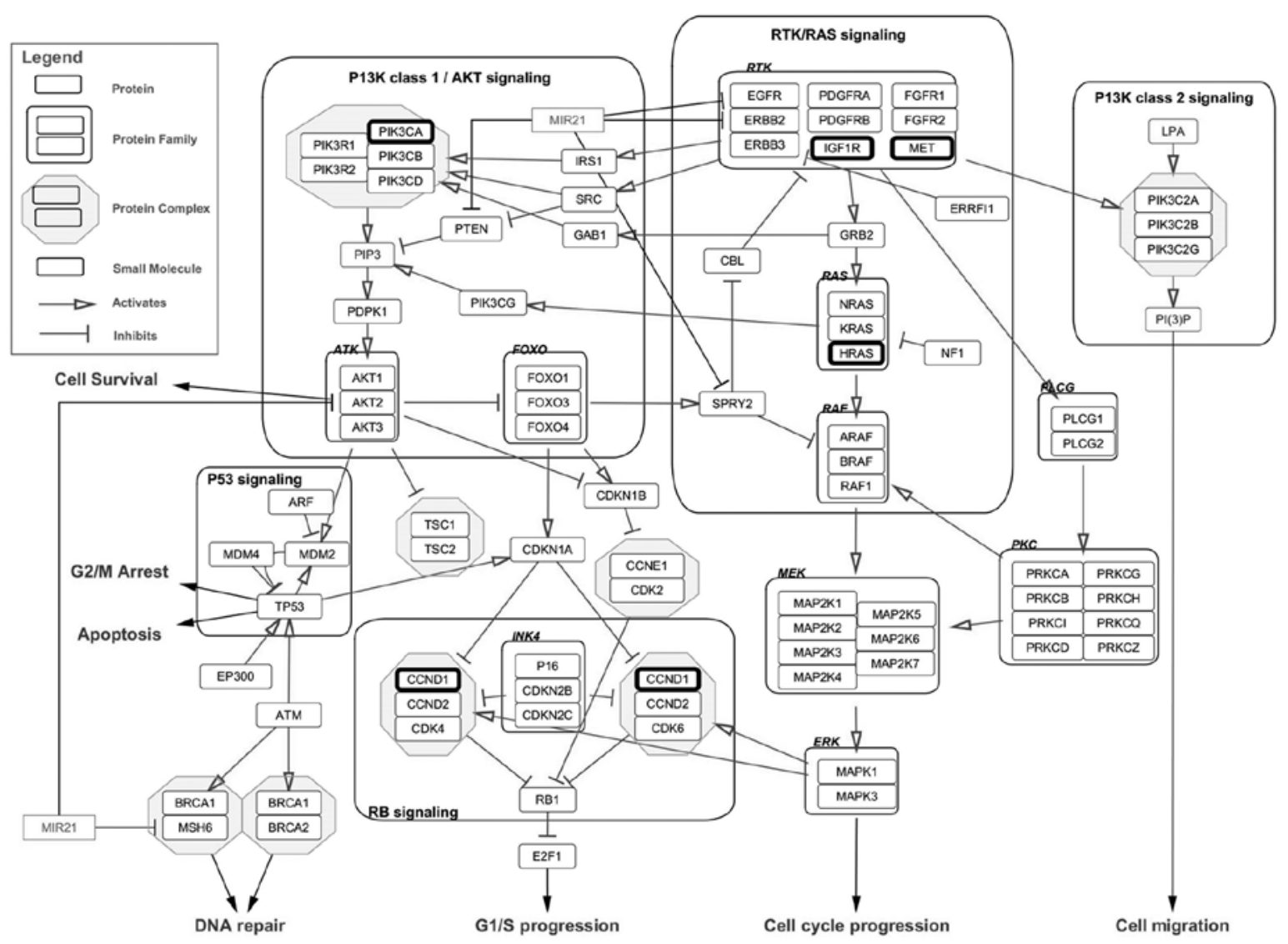

Figure 5. Pathway Map of Glioblastoma obtained from Wikipathways using Cytoscape. Targets genes of deregulated miRNAs are highlighted with thick borders. Five targets of downregulated miRNAs; CCND1, IGF1R, PIK3CA, HRAS and MET, found to play an important role in GBM signaling cascades. miRNAs, microRNAs; CCND1, cyclin D1; IGF1R, insulin like growth factor 1 receptor; PIK3CA, phosphatidylinositol-4,5-biphosphate 3-kinase catalytic subunit $\alpha$; HRAS, HRas proto-oncogene, GTPase; MET, MET proto-oncogene, receptor tyrosine kinase.

cascades (Fig. 5). Of the 6 miRNAs that were downregulated, the 2 miRNAs, hsa-miR-139-5p (IGF1R, PIK3CA, HRAS, MET) and hsa-miR-383-5p (CCND1, IGF1R), targeted all five of these genes. CCND1, IGF1R, PIK3CA and HRAS were also found to be present in the glioma pathways in KEGG database.

To identify the association between shortlisted target genes and survival of GBM patients, we used an online tool that correlates gene expression with patient survival from Cancer Genome Atlas (TCGA) data called OncoLnc. Patients were categorized into high expression and low expression groups based on 25th percentile cut off. Our analysis showed that high levels of HRAS expression predicted significantly less survival (Cox Coefficient $=0.155)$, with a Log-rank P-value $=0.0242$ in GBM patients (Fig. 6A). Similarly, higher expression of MET in GBM is correlated with poor patient survival (Cox Coefficient=0.136), with a Log-rank P-value $=0.0529$ (Fig. 6B). The three other genes (CCND1, IGF1R and PIK3CA), did not show any significant difference in the overall survival of GBM patients. Both HRAS and MET, which are key players in GBM disease progression, are the targets of hsa-miR-139-5p. Thus among the 6 miRNAs, down-regulation of hsa-miR-139-5p could play a very crucial role in the development of GBM and restoration of the normal expression levels of this miRNA can be further explored for therapeutic purposes to increase the overall survival of GBM patients.
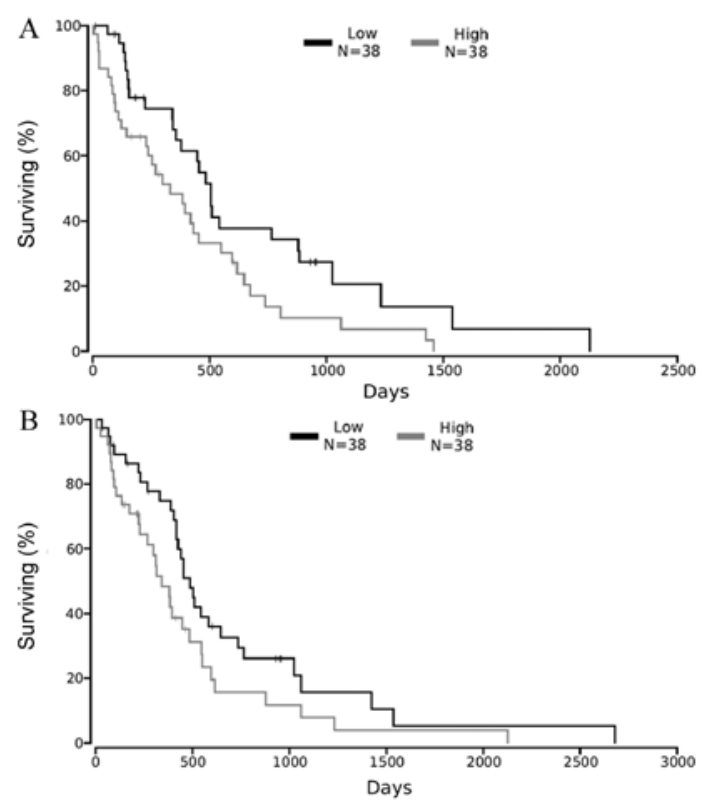

Figure 6. Survival analysis of target genes in the GBM pathway using an online tool OncoLnc that correlates gene expression data with patient survival from The Cancer Genome Atlas. (A) Survival analysis of HRAS. Elevated expression levels of HRAS is correlated with significant reduction in patient survival (Cox Coefficient=0.155, Log-rank P-value=0.0242). (B) Survival analysis of MET. Higher expression levels of MET associated with lower patient survival (Cox Coefficient=0.136, Log-rank P-value=0.0529). GBM, glioblastoma multiforme; HRAS, HRas proto-oncogene, GTPase; MET, MET proto-oncogene, receptor tyrosine kinase. 


\section{Discussion}

GBM is a very deadly type of brain cancer with reduced survival and a high degree of recurrence. Even though considerable efforts have been taken to find effective treatment strategies, there is no significant improvement observed with patient survival. Therefore there is an urgent need for identification of novel therapeutic approaches which require an understanding of the molecular markers that play an important role in the disease progression. Changes in the expression levels of miRNAs (which are endogenous regulators of tumour suppressor genes or oncogenes), play a critical role in cancer progression or development. Analysis of microarray expression data from GEO indicated 6 miRNAs to be down-regulated in both data sets studied. Instead of depending on the significance levels based on P-values for the identification of deregulated miRNAs, False Discovery Rate (FDR) was calculated on the basis of adjusted $\mathrm{p}$ values (Adj P-Values), which will reduce the probability of false positive result. This stringent statistical criteria along with a minimum 2 fold difference in expression, is the reason for the low number of deregulated miRNAs observed in the study. Furthermore, this methodology increases the confidence of the results for identification of reliable miRNA signatures in GBM, highly increasing the probability of obtaining specific miRNA patterns that are true positives.

Among the deregulated miRNAs, hsa-miR-338-5p, which showed the highest average downregulation, was already reported to be involved in the regulation of different types of cancers. In gastric cancers, hsa-miR-338-5p has been found to be downregulated and its overexpression in cell lines inhibits cell growth and proliferation by targeting BMI1 (12). Bioinformatics analysis has also identified hsa-miR-338-5p as a diagnostic target for hepatocellular carcinoma (HCC) (13). Furthermore, in GBM this miRNA was shown to sensitize cancer cells to radiation by targeting genes involved in DNA damage response (14). Studies have also demonstrated the ability of this miRNA to inhibit proliferation, suppress migration and invasion, and induce apoptosis in U87 GBM cell lines (15). Though there are no reports on the expression of hsa-miR-338-5p in different stages of GBM patients, a rise in the expression of hsa-miR-338-5p was correlated with increase in tumor stage in hepatocellular carcinoma (HCC). Reports of expression analysis based on tumor grade indicate that well differentiated tissues from HCC have higher expression levels of the miRNA as compared to poorly or moderately differentiated tumors (16).

Although there was a strong down-regulation of hsa-miR-138-2-3p in both the datasets, we were unable to find any target gene(s) for this miRNA using miRTarBase. There have been no previous studies thus far that implicate a role for this miRNA in GBM. However, this miRNA has been reported to play a critical role in laryngeal cancer by enhancing radio-sensitivity of laryngeal squamous cancer stem cells. Overexpression of this miRNA also decreases proliferation and invasion, induces cell cycle arrest, downregulates $\beta$-catenin in Wnt signaling pathway and enhances the expression of p38 and JNK1 in MAPK signaling pathway (17). Analysis of plasma samples from non-small cell lung cancer (NSCLC) patients with and without primary resistance to
Tyrosine Kinase Inhibitors (TKIs), showed a downregulation in the expression level of hsa-miR-138-2-3p in TKI resistant patients (18). Taken together, these observations suggest that this miRNA could also play an important role in GBM. Further analysis and focused studies are required to identify the target genes of this miRNA and its expression patterns in different stages of cancer in order to identify its effects on GBM.

Previous studies have shown downregulation of miRNA-770-5p in tumors associated with the brain such as gliomas (19) and medulloblastomas (MB) (20). Even amongst different types of brain cancers, the expression levels of hsa-miR-770-5p was significantly lower in MB as compared to astrocytomas (21). Our observations further support these studies by demonstrating down-regulation of hsa-miR-770-5p in brain tumors. There is no direct evidence suggesting a role for this miRNA in cancers of the central nervous system, but in HCC, Wnt/ $\beta$-catenin signaling inhibits the expression of FBXW7, which serves as a tumor suppressor, by inducing expression of hsa-miR-770-5p (22). This miRNA could also be a useful biomarker for predicting the chemoresistance towards cisplatin in ovarian cancer patients and was found to act as a tumor suppressor by downregulating ERCC2 expression (23). Reduced expression of hsa-miRNA-770-5p was observed in higher stages of glioma (WHO grade III and IV) as compared to lower stages (WHO grade I and II) (19).

Various studies have reported the role of hsa-miR-383-5p in the regulation of cell proliferation and tumor growth while upregulation of hsa-miR-383 was shown to induce $\mathrm{G}_{0} / \mathrm{G}_{1}$ phase arrest in glioma cells (24-26). MicroRNA-383 is reported to be downregulated in MB and overexpression of this miRNA promoted apoptosis in MB cells. In glioma patients the expression levels of this miRNA is negatively correlated with increase in the stage of the tumor (27). Our analysis also showed significant down-regulation of hsa-miR-383-5p in GBM samples, which is consistent with and corroborates the observations from previous studies showing decreased expression of miR-383 in gliomas as compared to normal brain tissues (25).

MicroRNA-139 is reported to play a significant role in different types of cancers such as gastric cancer (28), osteosarcomas (29), hepatocellular carcinoma (30), and papillary thyroid carcinoma (31). MicroRNA-139 was shown to inhibit proliferation and enhance apoptosis in temozolomide treated gliomas (32), and is reported to sensitize ovarian cancer cells to cisplatin chemotherapy (33). Specific studies on the patterns of hsa-miR-139 in different stages of GBM is absent, but a significant decrease in the expression of hsa-miR-139 was reported in HCC with advancement of the disease state. The metastatic tumor tissues expressed lower levels of hsa-miR-139 compared to their primary tumor tissues (34). There was a $60 \%$ reduction in hsa-miR-139 expression observed in metastatic lesions of laryngeal squamous carcinoma cells (LSCC), as compared to primary LSCC tissues (35). These observations along with reduced expression of hsa-miR-139 in GBM implies the possibility of similar expression profiles in different stages of GBM. MicroRNA-139 precursors could yield two mature miRNAs, hsa-miR-139-3p and hsa-miR-139-5p. Our study reported the down-regulation of both these mature miRNAs in GBM tissues. MicroRNA-139-3p has been reported to serve as a prognostic biomarker for bile duct cancer (36). Several studies 
have also indicated the possibility of using hsa-miR-139-3p as a diagnostic biomarker for colorectal cancer (37-40). Although there are no reports describing the role of hsa-miR-139-3p in glioblastoma, our study shows a significant change in expression levels of this miRNA in GBM tissues, suggesting a possible role in the development of GBM. Differences in hsa-miR-139-5p expression patterns have been described between primary and recurrent glioblastoma (36). Expression levels of miRNA-139-5p were also reported to predict as well as distinguish between long-term survivors and short-term survivors of GBM (41). Furthermore, miRNA-139-5p is also known to suppress the migration, invasion and proliferation of GBM cells $(42,43)$. Taken together, these findings confirm hsa-miR-139-5p to play a key role in GBM development and suggests its possible use as a biomarker for diagnosis of the disease as well as prediction of overall survival of the patients. Results from our analysis further support these observations that show a significant down-regulation of hsa-miR-139-5p in GBM (41,44).

Pathway analysis of target genes of deregulated miRNAs showed significant enrichment in pathways associated with cancer. Fourteen genes, namely, HRAS, ROCK2, MET, NFKB1, LPAR1, IGF1R, WNT1, FOS, CCND1, CXCR4, BCL2, JUN, VEGFA and PIK3CA were involved in pathways in cancer. The second most enriched pathway included focal adhesion, which plays a significant role in several important biological processes such as cell proliferation, differentiation and motility. An excessive activation of the PI3K-Akt signaling pathway in GBM induces cell proliferation, enhanced migratory potential and reduced survival of the patients $(45,46)$. Deregulation of PI3K-Akt signaling pathway is observed in a number of tumors including GBM. Phosphatase and Tensin homolog (PTEN) is a negative regulator of PI3K pathway, which dephosphorylates phosphoinositide, resulting in the termination of PI3K pathway signaling. The fact that around $70 \%$ of GBM patients harbor alterations in PTEN expression, shows the critical role of PI3K pathway in GBM $(47,48)$. PI3K pathway represses the PTEN transcription, both via activation of NFkB pathway and by inducing the expression of NEDD4-1, an E3 ligase involved in the ubiquitination of PTEN (48-51). In pediatric low-grade gliomas (pLGGs), microRNA-139-5p has been identified to target PIK3CA gene, which encodes the catalytic unit of PI3K (52). The possibility of additive regulation of PI3K pathway by PTEN and miR-139-5p in normal cells cannot be excluded. Down regulation of PI3K targeting miRNAs may shift the homeostasis of PI3K-PTEN regulatory network, which in turn results in PTEN down regulation, and further activation of PI3K pathway (48). In PTEN negative GBM, down regulation of miRNAs that target PI3K pathway might lead to hyper activation of PI3K, resulting in an increase in aggressiveness of cancer. It is not yet determined if the loss or alteration in PTEN accounts for the down regulation of $\mathrm{PI} 3 \mathrm{~K}$ regulating miRNA, or whether the alteration in miRNA expression results in altered PTEN expression. The association of PTEN and miRNAs regulating PI3K needs to be further evaluated in GBM. Prolactin signaling pathway, another pathway enriched with target genes is found to play dynamic roles in both colorectal cancer (53) and breast cancer (54). Nine targets genes were found to be associated with the HTLV-I infection pathway, which leads to adult T-cell leukemia (ATL). BMI1, NOTCH1, HRAS, CCND1, MCL1, BCL2, VEGFA, MET and NFKB1 were enriched in 'MicroRNAs in cancer pathway'. Other significantly enriched pathways included, renal cell carcinoma, prostate cancer and hepatitis B. Amongst the target genes enriched in different pathways, NFKB1 is found to be associated with many types of human cancers including $\operatorname{GBM}(55,56)$ and is known to be elevated in glioma cells (57). Overexpression of NFKB1 inhibited the apoptosis of glioma cells by inducing the BCL2 expression. The over expression of BCL2, an anti-apoptotic gene, highly expressed in majority of GBM tissues, is associated with increased resistance to anti-cancer drugs (58). Inhibition of BCL2 expression in colorectal cancer cells by hsa-miR-139-5p reduced the epithelial to mesenchymal transition and improved chemo sensitivity (59). Studies on esophageal squamous cell carcinoma showed positive regulation of MCL1 by NFKB (60). MCL1 is an oncogene and is a member of BCL2 gene family which is highly expressed in GBM. MCL1 inhibition in GBM showed induction of apoptosis and increased chemo sensitivity (61). All these observations show the critical role of deregulated miRNAs identified in this study, in the regulation of GBM development and progression. Protein-protein interaction (PPI) network of targets genes predicted significant levels of interaction among them and the only single cluster identified from the network had eight nodes, with VEGFA as the seed gene. VEGFA is known to play a vital role in a wide variety of cancers, promoting angiogenesis and inducing cell survival.

Mapping of target genes to the glioblastoma pathway revealed five genes namely CCND1, IGF1R, PIK3CA, HRAS and MET as a part of the pathway reiterating the importance of deregulated miRNAs in the regulation of GBM. hsa-miR-139-5p is known to target genes such as IGF1R, PIK3CA, HRAS and MET, which might play a very critical role in the development of GBM. Previous studies have reported that increased levels of hsa-miR-139-5p showed a significant improvement in the overall survival of the GBM patients (41). Concurrent with these observations, the current study also demonstrated that lowered expression levels of hsa-miR-139-5p target genes like HRAS and MET, predicted increased survival in GBM patients.

In conclusion, we have identified differentially expressed miRNAs in GBM tissues from miRNA expression profiles, downloaded from GEO. Our analysis identified six downregulated miRNAs and their targets that might play a crucial role in GBM, including HRAS and MET. Furthermore, survival analysis showed that hsa-miR-139-5p could play a significant role in the overall survival of GBM patients and restoring the levels of this miRNA in GBM tissues might show a therapeutic advantage in the treatment of the disease. Further studies are needed for confirmation of our observations, but this study could provide the basis for understanding the roles of these miRNAs in GBM.

\section{Acknowledgements}

The authors would like to thank Dr Mata Amritanandamayi (Amrita Vishwa Vidyapeetham) for guidance in the preparation of the manuscript. 


\section{Funding}

The University Grants Commission (grant no. BININ00357398) provided financial assistance in the form of a fellowship to SS. The present study was supported by institutional funding (Amrita Vishwa Vidyapeetham; grant no. AM/ASBT/RE-03/2017).

\section{Availability of data and materials}

The datasets generated and/or analyzed during the presemt study are available in the Gene Expression Omnibus repository, https://www.ncbi.nlm.nih.gov/geo.

\section{Authors' contributions}

BN, GK and SS conceived and designed the study. SS, DS and NM acquired data. SS and DS performed the experiments and analyzed the data. BN, GK and SS wrote the manuscript. All authors read and approved the final manuscript. All authors read and approved the manuscript and agree to be accountable for all aspects of the research in ensuring that the accuracy or integrity of any part of the work are appropriately investigated and resolved.

\section{Ethics approval and consent to participate}

Not applicable.

\section{Patient consent for publication}

Not applicable.

\section{Competing interests}

The authors declare that they have no competing interests.

\section{References}

1. Ramachandran R, Junnuthula VR, Gowd GS, Ashokan A, Thomas J, Peethambaran R, Thomas A, Unni AK, Panikar D, Nair SV and Koyakutty M: Theranostic 3-Dimensional nano brain-implant for prolonged and localized treatment of recurrent glioma. Sci Rep 7: 43271, 2017.

2. Malhotra M, Sekar TV, Ananta JS, Devulapally R, Afjei R, Babikir HA, Paulmurugan R and Massoud TF: Targeted nanoparticle delivery of therapeutic antisense microRNAs presensitizes glioblastoma cells to lower effective doses of temozolomide in vitro and in a mouse model. Oncotarget 9: 21478-21494, 2018.

3. Wong ET, Hess KR, Gleason MJ, Jaeckle KA, Kyritsis AP, Prados MD, Levin VA and Yung WK: Outcomes and prognostic factors in recurrent glioma patients enrolled onto phase II clinical trials. J Clin Oncol 17: 2572-2578, 1999.

4. Lamborn KR, Yung WK, Chang SM, Wen PY, Cloughesy TF, DeAngelis LM, Robins HI, Lieberman FS, Fine HA, Fink KL, et al: Progression-free survival: An important end point in evaluating therapy for recurrent high-grade gliomas. Neuro Oncol 10: 162-170, 2008.

5. Schmalz PG, Shen MJ and Park JK: Treatment resistance mechanisms of malignant glioma tumor stem cells. Cancers (Basel) 3: 621-635, 2011

6. Lee RC, Feinbaum RL and Ambros V: The C. Elegans heterochronic gene lin- 4 encodes small RNAs with antisense complementarity to lin-14. Cell 75: 843-854, 1993.

7. Calin GA, Dumitru CD, Shimizu M, Bichi R, Zupo S, Noch E, Aldler H, Rattan S, Keating M, Rai K, et al: Frequent deletions and down-regulation of micro-RNA genes miR15 and miR16 at $13 q 14$ in chronic lymphocytic leukemia. Proc Natl Acad Sci USA 99: 15524-15529, 2002.
8. Khan AA, Advani J, Patel K, Nanjappa V, Datta KK, Solanki HS, Kumar P, Mathur PP, Nair B, Keshava Prasad TS, et al: Chronic exposure to cigarette smoke and chewing tobacco alters expression of microRNAs in esophageal epithelial cells. Microrna 7: 28-37, 2018.

9. Cai Y, Yu X, Hu S and Yu J: A brief review on the mechanisms of miRNA regulation. Genomics Proteomics Bioinformatics 7: 147-154, 2009.

10. Zhang B, Pan X, Cobb GP and Anderson TA: microRNAs as oncogenes and tumor suppressors. Dev Biol 302: 1-12, 2007.

11. Reddy KB: MicroRNA (miRNA) in cancer. Cancer Cell Int 15: $38,2015$.

12. Tong D, Zhao L, He K, Sun H, Cai D, Ni L, Sun R, Chang S, Song $\mathrm{T}$ and Huang C: MECP2 promotes the growth of gastric cancer cells by suppressing miR-338-mediated antiproliferative effect. Oncotarget 7: 34845-34859, 2016.

13. Liang L, Gao L, Zou XP, Huang ML, Chen G, Li JJ and Cai XY: Diagnostic significance and potential function of miR-338-5p in hepatocellular carcinoma: A bioinformatics study with microarray and RNA sequencing data. Mol Med Rep 17: 2297-2312, 2018.

14. Besse A, Sana J, Lakomy R, Kren L, Fadrus P, Smrcka M, Hermanova M, Jancalek R, Reguli S, Lipina R, et al: MiR-338-5p sensitizes glioblastoma cells to radiation through regulation of genes involved in DNA damage response. Tumour Biol 37: 7719-7727, 2016.

15. Lei D, Zhang F, Yao D, Xiong N, Jiang X and Zhao H: MiR-338-5p suppresses proliferation, migration, invasion, and promote apoptosis of glioblastoma cells by directly targeting EFEMP1. Biomed Pharmacother 89: 957-965, 2017.

16. Huang XH, Wang Q, Chen JS, Fu XH, Chen XL, Chen LZ, Li W, Bi J, Zhang LJ, Fu Q, et al: Bead-based microarray analysis of microRNA expression in hepatocellular carcinoma: miR-338 is downregulated. Hepatol Res 39: 786-794, 2009.

17. Zhu Y, Shi LY,Lei YM, Bao YH,LiZY,Ding F,Zhu GT, Wang QQ and Huang CX: Radiosensitization effect of hsa-miR-138-2-3p on human laryngeal cancer stem cells. PeerJ 5: e3233, 2017.

18. Ma Y, Pan X, Xu P, Mi Y, Wang W, Wu X, He Q, Liu X, Tang W and An HX: Plasma microRNA alterations between EGFR-activating mutational NSCLC patients with and without primary resistance to TKI. Oncotarget 8: 88529-88536, 2017.

19. Zhang JF, Zhang JS, Zhao ZH, Yang PB, Ji SF, Li N, Shi QD, Tan J, Xu X, Xu CB and Zhao LY: MicroRNA-770 affects proliferation and cell cycle transition by directly targeting CDK8 in glioma. Cancer Cell Int 18: 195, 2018.

20. Dai J, Li Q, Bing Z, Zhang Y, Niu L, Yin H, Yuan G and Pan Y: Comprehensive analysis of a microRNA expression profile in pediatric medulloblastoma. Mol Med Rep 15: 4109-4115, 2017.

21. Eguia-AguilarP,Gutierrez-CastilloLandPerezpena-DiazcontiM: Expression of microRNAs in tumors of the central nervous system in pediatric patients in México. Childs Nerv Syst 33: 2117-2128, 2017.

22. Wu WJ, Shi J, Hu G, Yu X, Lu H, Yang ML, Liu B and Wu ZX: Wnt/ $\beta$-catenin signaling inhibits FBXW7 expression by upregulation of microRNA-770 in hepatocellular carcinoma. Tumour Biol 37: 6045-6051, 2016.

23. Zhao H, Yu X, Ding Y, Zhao J, Wang G, Wu X, Jiang J, Peng C, Guo GZ and Cui S: MiR-770-5p inhibits cisplatin chemoresistance in human ovarian cancer by targeting ERCC2. Oncotarget 7: 53254-53268, 2016.

24. Lian J, Tian H, Liu L, Zhang XS, Li WQ, Deng YM, Yao GD, Yin MM and Sun F: Downregulation of microRNA-383 is associated with male infertility and promotes testicular embryonal carcinoma cell proliferation by targeting IRF1. Cell Death Dis 1: e94, 2010

25. Xu Z, Zeng X, Tian D, Xu H, Cai Q, Wang J and Chen Q: MicroRNA-383 inhibits anchorage-independent growth and induces cell cycle arrest of glioma cells by targeting CCND1. Biochem Biophys Res Commun 453: 833-838, 2014.

26. Lü M, Tian H, Cao YX, He X, Chen L, Song X, Ping P, Huang H and Sun F: Downregulation of miR-320a/383-sponge-like long non-coding RNA NLC1-C (narcolepsy candidate-region 1 genes) is associated with male infertility and promotes testicular embryonal carcinoma cell proliferation. Cell Death Dis 6: e1960, 2015.

27. Xu D, Ma P, Gao G, Gui Y, Niu X and Jin B: MicroRNA-383 expression regulates proliferation, migration, invasion, and apoptosis in human glioma cells. Tumour Biol 36: 7743-7753, 2015.

28. Yu X, Ma C, Fu L, Dong J and Ying J: MicroRNA-139 inhibits the proliferation, migration and invasion of gastric cancer cells by directly targeting $\mathrm{Q}$-associated protein kinase 1 . Oncol Lett 15: 5977-5982, 2018. 
29. Su S and Nie X: MiR-139 prompts the development of osteosarcomas mainly through targeting ROCK1. Pharmazie 72: 759-763, 2017.

30. Zhang P, Yin J, Yuan L, Bai Q and Lu J: microRNA-139 suppresses proliferation of hepatocellular carcinoma cells by silencing of B cell translocation gene 3. Xi Bao Yu Fen Zi Mian Yi Xue Za Zhi 33: 1516-1520, 2017 (In Chinese).

31. Ye Y, Zhuang J, Wang G, He S, Ni J and Xia W: MicroRNA-139 targets fibronectin 1 to inhibit papillary thyroid carcinoma progression. Oncol Lett 14: 7799-7806, 2017.

32. Li RY, Chen LC, Zhang HY, Du WZ, Feng Y, Wang HB, Wen JQ, Liu X, Li XF, Sun Y, et al: MiR-139 inhibits Mcl-1 expression and potentiates TMZ-induced apoptosis in glioma. CNS Neurosci Ther 19: 477-483, 2013

33. Xiao F, Li Y, Wan Y and Xue M: MircroRNA-139 sensitizes ovarian cancer cell to cisplatin-based chemotherapy through regulation of ATP7A/B. Cancer Chemother Pharmacol 81: 935-947, 2018.

34. Wong CC, Wong CM, Tung EK, Au SL, Lee JM, Poon RT, Man K and Ng IO: The microRNA miR-139 suppresses metastasis and progression of hepatocellular carcinoma by down-regulating Rho-kinase 2. Gastroenterology 140: 322-331, 2011.

35. Luo HN, Wang ZH, Sheng Y, Zhang Q, Yan J, Hou J, Zhu K, Cheng Y, Xu YL, Zhang XH, et al: MiR-139 targets CXCR4 and inhibits the proliferation and metastasis of laryngeal squamous carcinoma cells. Med Oncol 31: 789, 2014.

36. Bo LJ, Wei B, Li ZH, Wang ZF, Gao Z and Miao Z: Bioinformatics analysis of miRNA expression profile between primary and recurrent glioblastoma. Eur Rev Med Pharmacol Sci 19: 3579-3586, 2015.

37. Lin M, Chen W, Huang J, Gao H, Ye Y, Song Z and Shen X: MicroRNA expression profiles in human colorectal cancers with liver metastases. Oncol Rep 25: 739-747, 2011.

38. Kanaan Z, Roberts H, Eichenberger MR, Billeter A, Ocheretner G, Pan J, Rai SN, Jorden J, Williford A and Galandiuk S: A plasma microRNA panel for detection of colorectal adenomas: A step toward more precise screening for colorectal cancer. Ann Surg 258: 400-408, 2013.

39. Chen WC, Lin MS, Ye YL, Gao HJ, Song ZY and Shen XY: microRNA expression pattern and its alteration following celecoxib intervention in human colorectal cancer. Exp Ther Med 3: 1039-1048, 2012

40. Liu X, Duan B, Dong Y, He C, Zhou H, Sheng H, Gao H and Zhang X: MicroRNA-139-3p indicates a poor prognosis of colon cancer. Int J Clin Exp Pathol 7: 8046-8052, 2014.

41. Yuan GQ, Wei NL, Mu LY, Wang XQ, Zhang YN, Zhou WN and Pan YW: A 4-miRNAs signature predicts survival in glioblastoma multiforme patients. Cancer Biomark 20: 443-452, 2017.

42. Yue S, Wang L, Zhang H, Min Y, Lou Y, Sun H, Jiang Y, Zhang W, Liang A, Guo Y, et al: miR-139-5p suppresses cancer cell migration and invasion through targeting ZEB1 and ZEB2 in GBM. Tumour Biol 36: 6741-6749, 2015.

43. Dai S, Wang X, Li X and Cao Y: MicroRNA-139-5p acts as a tumor suppressor by targeting ELTD1 and regulating cell cycle in glioblastoma multiforme. Biochem Biophys Res Commun 467: 204-210, 2015

44. Sana J, Radova L, Lakomy R, Kren L, Fadrus P, Smrcka M, Besse A, Nekvindova J, Hermanova M, Jancalek R, et al: Risk Score based on microRNA expression signature is independent prognostic classifier of glioblastoma patients. Carcinogenesis 35: 2756-2762, 2014

45. Westhoff MA, Karpel-Massler G, Brühl O, Enzenmüller S, La Ferla-Brühl K, Siegelin MD, Nonnenmacher L and Debatin KM: A critical evaluation of PI3K inhibition in glioblastoma and neuroblastoma therapy. Mol Cell Ther 2: 32, 2014.

46. Langhans J, Schneele L, Trenkler N, von Bandemer H, Nonnenmacher L, Karpel-Massler G, Siegelin MD, Zhou S, Halatsch ME, Debatin KM and Westhoff MA: The effects of PI3K-mediated signalling on glioblastoma cell behaviour. Oncogenesis 6: 398, 2017.
47. Xu J, Li Z, Wang J, Chen $\mathrm{H}$ and Fang JY: Combined PTEN mutation and protein expression associate with overall and disease-free survival of glioblastoma patients. Transl Oncol 7: 196-205.e1, 2014

48. Carracedo A and Pandolfi PP: The PTEN-PI3K pathway: Of feedbacks and cross-talks. Oncogene 27: 5527-5541, 2008

49. Vasudevan KM, Gurumurthy S and Rangnekar VM: Suppression of PTEN expression by NF-kappa B prevents apoptosis. Mol Cell Biol 24: 1007-1021, 2004.

50. Wang X, Trotman LC, Koppie T, Alimonti A, Chen Z, Gao Z, Wang J, Erdjument-Bromage H, Tempst P, Cordon-Cardo C, et al: NEDD4-1 is a proto-oncogenic ubiquitin ligase for PTEN. Cell 128: 129-139, 2007.

51. Ahn Y, Hwang CY, Lee SR, Kwon KS and Lee C: The tumour suppressor PTEN mediates a negative regulation of the E3 ubiquitin-protein ligase Nedd4. Biochem J 412: 331-338, 2008.

52. Catanzaro G, Besharat ZM, Miele E, Chiacchiarini M, Po A, Carai A, Marras CE, Antonelli M, Badiali M, Raso A, et al: The miR-139-5p regulates proliferation of supratentorial paediatric low-grade gliomas by targeting the PI3K/AKT/mTORC1 signalling. Neuropathol Appl Neurobiol 44: 687-706, 2018

53. Neradugomma NK, Subramaniam D, Tawfik OW, Goffin V, Kumar TR, Jensen RA and Anant S: Prolactin signaling enhances colon cancer stemness by modulating Notch signaling in a Jak2-STAT3/ERK manner. Carcinogenesis 35: 795-806, 2014.

54. Tworoger SS and Hankinson SE: Prolactin and breast cancer etiology: An epidemiologic perspective. J Mammary Gland Biol Neoplasia 13: 41-53, 2008.

55. Yang TQ, Chen M, Wang YQ, Xu W, Han Y, Xu J, Xiang YJ, Yuan B, Wang HZ and Zhou YX: Nuclear factor-kappa B1 inhibits early apoptosis of glioma cells by promoting the expression of Bcl-2. Onco Targets Ther 10: 4305-4313, 2017.

56. Kina I, Sultuybek GK, Soydas T, Yenmis G, Biceroglu H, Dirican A, Uzan M and Ulutin T: Variations in Toll-like receptor and nuclear factor-kappa $\mathrm{B}$ genes and the risk of glioma. Br J Neurosurg 33: 165-170, 2019.

57. Hayashi S, Yamamoto M, Ueno Y, Ikeda K, Ohshima K, Soma G and Fukushima T: Expression of nuclear factor-kappa B, tumor necrosis factor receptor type 1 , and c-Myc in human astrocytomas. Neurol Med Chir (Tokyo) 41: 187-195, 2001.

58. Fels C, Schäfer C, Hüppe B, Bahn H, Heidecke V, Kramm CM, Lautenschläger $\mathrm{C}$ and Rainov NG: $\mathrm{Bcl}-2$ expression in higher-grade human glioma: A clinical and experimental study. J Neurooncol 48: 207-216, 2000.

59. Li Q, Liang X, Wang Y, Meng X, Xu Y, Cai S, Wang Z, Liu J and Cai G: miR-139-5p inhibits the epithelial-mesenchymal transition and enhances the chemotherapeutic sensitivity of colorectal cancer cells by downregulating BCL2. Sci Rep 6: 27157, 2016.

60. Liu H, Yang J, Yuan Y, Xia Z, Chen M, Xie L, Ma X, Wang J, Ouyang S, Wu Q, et al: Regulation of Mcl-1 by constitutive activation of NF- $\kappa \mathrm{B}$ contributes to cell viability in human esophageal squamous cell carcinoma cells. BMC Cancer 14: 98, 2014.

61. Day BW, Stringer BW, Spanevello MD, Charmsaz S, Jamieson PR Ensbey KS, Carter JC, Cox JM, Ellis VJ, Brown CL, et al: ELK4 neutralization sensitizes glioblastoma to apoptosis through downregulation of the anti-apoptotic protein Mcl-1. Neuro Oncol 13: 1202-1212, 2011.

This work is licensed under a Creative Commons Attribution-NonCommercial-NoDerivatives 4.0 International (CC BY-NC-ND 4.0) License. 